\title{
ATR-FTIR spectroscopic study of the effect of ceramic addition in novel ionoconductor gels for biomedical applications in space
}

\author{
Paola Romagnoli ${ }^{\mathrm{a}, *}$, Marcella Trombetta ${ }^{\mathrm{b}}$, Silvia Licoccia ${ }^{\mathrm{a}}$ \\ a Dipartimento di Scienze e Tecnologie Chimiche, Università di Roma Tor Vergata, Via della Ricerca Scientifica 1, 00133 Rome, Italy \\ ' Interdisciplinary Center for Biomedical Research (CIR), Laboratory of Biomaterials, Università "Campus Bio-Medico", \\ Via E. Longoni 83, 00155 Rome, Italy
}

\begin{abstract}
The preparation and characterization of composite polymer electrolyte membranes consisting of selected nanosized ceramic powders $\left(\mathrm{TiO}_{2}, \mathrm{Al}_{2} \mathrm{O}_{3}, \mathrm{SiO}_{2}\right)$ dispersed into poly(methylmethacrylate) $\mathrm{PMMA-based} \mathrm{gels} \mathrm{with} \mathrm{LiClO}_{4}$ and 1,2-diethoxyethane have been investigated. The electrical conductivity values, measured by a.c. impedance spectroscopy, demonstrated that the addition of the ceramic filler to the membranes contributes to enhance the electrochemical stability without significantly affecting their conductivity. The variations observed in conductivity have been correlated with the structural variations introduced by the presence of different ceramics evidentiated by ATR-FTIR spectroscopy. Preliminary measurements of bioelectrical signals (electroencephalograms, EEG) carried out with the newly prepared composite membranes showed they are suitable for neurological recording.

(C) 2003 Published by Elsevier Ltd.
\end{abstract}

Keywords: Biomedical application; Composites; Ionic conductivity; Membranes; Spectroscopy

\section{Introduction}

The development of new techniques for monitoring brain functionality directly in space, has received significant attention due to the need of studying the possible temporary and/or permanent altered function of astronauts' brains during long space missions. ${ }^{1}$ Our research has been directed to the development of ionoconducting solid gels to be used as an interface between the skin and the actual electrical instrumentation used to produce an electroencephalogram (EEG). Conventional EEG registrations are carried out using liquid or semi-fluid electrolytes to establish contact between the scalp and the electrical instrumentation. The skin must be accurately cleaned to optimize the measurement. Both skin cleaning and the use of fluid electrolytes represent major drawbacks if the EEG has to be recorded in space where astronaut's time is highly valued. We have previously reported on the preparation, electrochemical characterization and application in EEG recording of $\mathrm{Li}^{+}$-containing PMMA based membranes. These gels showed high and stable conductivity values

\footnotetext{
* Corresponding author.
}

and almost identical electrical responses in EEG measurement to those determined with conventional systems. ${ }^{2}$

To improve the performances of these materials a new synthetic procedure has been developed by varying the solvents used in the gel forming process and adding different nanometric ceramic powders $\left(\mathrm{TiO}_{2}, \mathrm{Al}_{2} \mathrm{O}_{3}\right.$, $\mathrm{SiO}_{2}$ ). The combination of different components in composite materials may in fact lead to the combination of the advantages of the single phases, and has been used in several applications ranging from aerospace technology, to the automotive industry, and medical technology. ${ }^{3-5}$

The new composite membranes have been electrochemically characterized and the interactions between their different components have been investigated by ATR-FTIR spectroscopy.

\section{Experimental}

All reagents (Aldrich) were reagent grade and were used without further purification. The particle size of oxide powders was certified from the producer and was $5.5 \mathrm{~nm}$ for $\mathrm{SiO}_{2}$ and $5.8 \mathrm{~nm}$ for $\mathrm{Al}_{2} \mathrm{O}_{3}$. Titania powders 
were prepared via sol-gel according to a procedure previously reported. ${ }^{6,7}$ The XRD pattern of the powder calcinated at $500{ }^{\circ} \mathrm{C}$ showed the presence of a single phase (anatase). The average particle size was determined to be $12 \mathrm{~nm}$ by application of the Scherrer's equation to peak $(1,0,1)$ broadening.

The electrolyte gels were prepared using the solvent casting technique. Typical preparation, described for a representative composite sample, is as follows: $\mathrm{LiClO}_{4}$ was dissolved in 1,2-diethoxyethane. PMMA and the ceramic component were then added, along with $\mathrm{EtOH} /$ $\mathrm{H}_{2} \mathrm{O} 9: 1$, the latter to reduce the viscosity of the mixture. Heating to $70{ }^{\circ} \mathrm{C}$ afforded complete gelification. The resulting gel was poured on a glass plate and cooled at RT $\left(22-25{ }^{\circ} \mathrm{C}\right)$ until the volatile casting solvent was completely evaporated favouring cross-linking and solid gel formation. The membranes had an average thickness of $1 \mathrm{~mm}$ and were stored in closed vials at room temperature.

Four membranes have been prepared: M1, to be used as reference, contained no ceramic powder, M2, M3 and $\mathrm{M} 4$ contained $5 \mathrm{wt} \% \mathrm{TiO}_{2}, \mathrm{Al}_{2} \mathrm{O}_{3}$ and $\mathrm{SiO}_{2}$ respectively. $\mathrm{Li}^{+}$concentration was kept constant at $0.8 \mathrm{M}$. The molar composition (\%) was 7.19-64.01-28.80 $\left(\mathrm{Li}^{+}{ }_{-}\right.$glyPMMA).

Disks having diameters of ca. $1 \mathrm{~cm}$ were cut from the membranes with a surgical blade to perform electrical and spectroscopic measurements. Homogeneity of the membranes and reproducibility of the preparation procedure were verified by performing measurements on different parts of the same membrane and on membranes deriving from different preparations.

Ionic conductivity was measured by impedance spectroscopy performed on teflon cells formed by sandwiching the given membranes between two stainless steel electrodes, which acted as blocking electrodes for the ions. The measurements were carried out using a Solartron frequency response analyzer (model 1260) scanning over $1 \mathrm{~Hz}-100 \mathrm{kHz}$. ATR-FTIR analysis have been performed using the Golden Gate MK II single reflection diamond ATR systems, Specac mounted on a spectrophotometer FT-IR Nexus 870 E.S.P., Nicolet. Membranes samples have been analyzed directly on an ATR platform. Bipolar EEG recordings (subject with eyes closed) were simultaneously obtained from conventional electrodes and the new solid electrolyte gels directly in contact with the scalp.

\section{Results and discussion}

The composition and conductivity values of the new composite membranes are listed in Table 1. Room temperature conductivities were greater than $10^{-3} \mathrm{~S} / \mathrm{cm}^{-1}$ for all four electrolytes prepared. The small variations can be directly related to the extent of surface inter-
Table 1

Conductivity of gel electrolytes having molar composition $\mathrm{Li}^{+}$:gly:PMMA = 1.0:8.9:4.0, doped with different ceramic oxides

\begin{tabular}{lll}
\hline $\begin{array}{l}\text { Membrane } \\
\text { label }\end{array}$ & $\begin{array}{l}\text { Ceramic filler } \\
(5 \mathrm{wt} \%)\end{array}$ & $\begin{array}{l}\text { Conductivity } \\
\left(\mathrm{S} \mathrm{cm}^{-1}\right)^{\mathrm{a}}\end{array}$ \\
\hline M1 & - & $3.28 \times 10^{-3}$ \\
M2 & $\mathrm{TiO}_{2}$ & $2.70 \times 10^{-3}$ \\
M3 & $\mathrm{Al}_{2} \mathrm{O}_{3}$ & $1.47 \times 10^{-3}$ \\
M4 & $\mathrm{SiO}_{2}$ & $2.90 \times 10^{-3}$ \\
\hline
\end{tabular}

a Data determined by a.c. impedance within $24 \mathrm{~h}$ from preparation.

action of the specific ceramic. Indeed, the variations in transport properties in the different composites are related to the occurrence of hydrogen bonds between the ceramics' surface and polymer chains and/or interactions with the lithium salt.

Although the presence of the ceramic fillers causes a reduction of the initial conductivity of the membranes, it highly improves their electrochemical stability.

A plot of conductance vs storage time is shown in Fig. 1, where a comparison is made between the time evolution of conductivity of PMMA-based polymer electrolytes with and without nanosized fillers. While the conductivity of the composite gels does not appreciably change upon long storage time, that of the ceramic-free membrane decays by about two orders of magnitude. It may be assumed that the enhancement of the mechanical stability is associated with the entangling of the polymer chains with the ceramic fillers. Furthermore, the liquid retention tendency of these particles may help in holding the electrolyte liquid component within the membrane structure. ${ }^{8}$

Thus, the well known water retention ability of ceramic oxides is cleary reflected by the highest stability in time of conductivity.

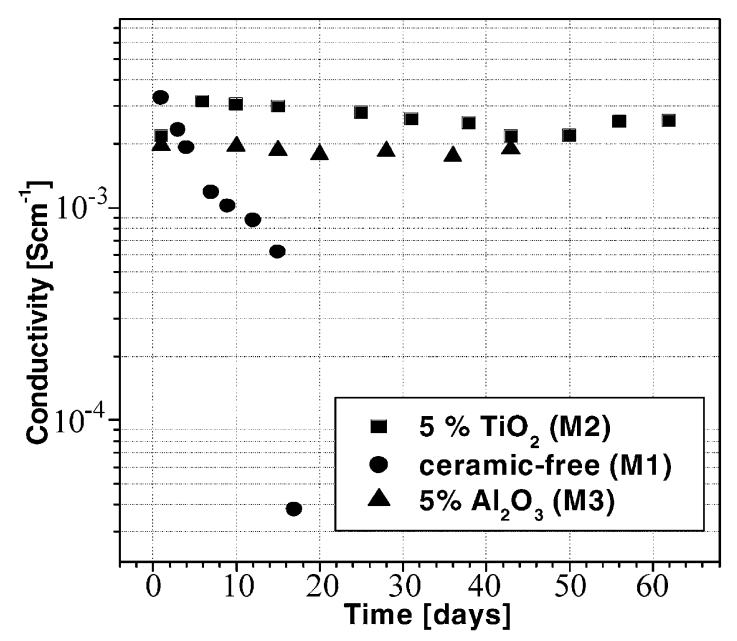

Fig. 1. Time evolution of the conductivity of PMMA-Gly$\mathrm{LiClO}_{4}+5 \% \mathrm{X}$ composites (M2, M3) and of the ceramic free membrane (M1). Data obtained by impedance spectroscopy. 
The influence of the addition of different ceramic fillers to the membranes has been investigated by means of ATR-FTIR spectroscopy. ATR-FTIR showed that different microstructural interactions occur varying the type of the ceramics' surface states. Fig. 2 shows the ATR-FTIR spectra of samples M1-M4 in the OHs stretching region. The spectra are characterized by three main components centered at about 3540, 3425 and $3290 \mathrm{~cm}^{-1}$. In the spectrum of the reference membrane M1 these bands can be attributed to the vibration of free water $\mathrm{OH}$ groups, water $\mathrm{H}$-bonded with carbonyl groups and to intermolecular $\mathrm{H}$-bonded water, respectively.

It can be observed that the relative intensity of the high frequency band decreases in the presence of ceramic fillers, while the second component $\left(3425 \mathrm{~cm}^{-1}\right)$ becomes predominant. This can be explained by the fact that in the presence of ceramics new H-bonds are generated between the $\mathrm{OH}$ groups on the surface and both the polymer carbonyl groups and water. We have to point out that the increase of the $3540 \mathrm{~cm}^{-1}$ absorption, more evident in the case of the titania containing sample (M2) with respect to the alumina containing composite membrane (M3), is due to the contribution of free $\mathrm{OHs}$ present on the oxide surfaces. In the case of the $\mathrm{SiO}_{2}$ containing sample (M4) surface hydroxyls ${ }^{9}$ are almost absent, as indicated by the decrease in intensity of the high frequency band.

Fig. 3 reports ATR/FTIR spectra of the different membranes in the $\mathrm{C}=\mathrm{O}$ stretching region. Two main bands, centered at 1728 and $1645 \mathrm{~cm}^{-1}$, characterize these spectra. The first band can be assigned to the combination of $\mathrm{vC}=\mathrm{O}$ of carbonyl groups electrostatically interacting within the polymer chain and with $\mathrm{Li}^{+}$ions, ${ }^{10}$ while the second one is due to $\delta \mathrm{O}-\mathrm{H}$ of water physically entrapped in the system and interacting through H-bonds. Comparison between the reference spectrum, corresponding to the ceramic free sample (M1, trace b), with the spectra measured for the composite membranes, shows that the intensity of the

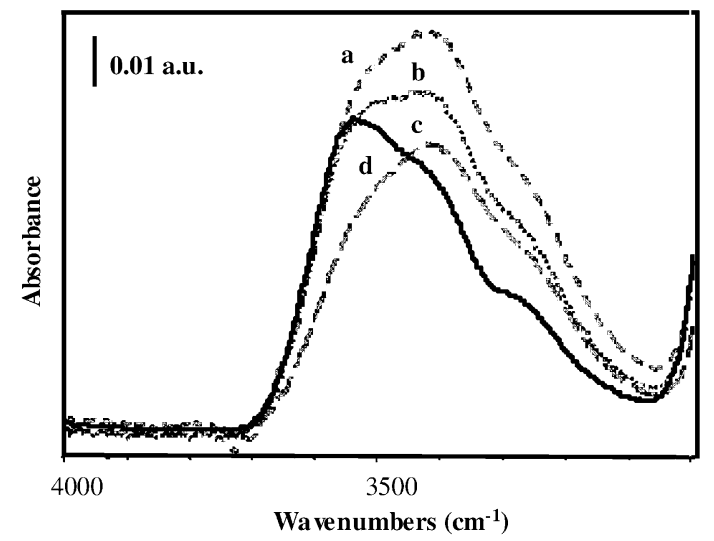

Fig. 2. ATR-FTIR spectra in the $\mathrm{OH}$ stretching region of membranes containing $\mathrm{LiClO}_{4} 0.8 \mathrm{M}$ doped with different ceramics: (a) M2, (b) M3, (c) M1 and (d) M4. Labels refer to Table 1.
$1728 \mathrm{~cm}^{-1}$ band decreases in the presence of the different ceramic fillers, demonstrating that the polymer carbonyl groups are involved in interactions with the ceramic phase. Variations in spectral intensities of the second band $\left(1645 \mathrm{~cm}^{-1}\right)$ are almost negligible for the titania and allumina containing samples (spectra $a$ and $c$, in Fig. 3, samples M2, M3) with respect to sample M1, indicating that the amount of crystallization water chemically entrapped in these gels is constant. Instead, a significant difference is observed for sample M4. The decrease in the IR activity of this band for the silica containing membrane indicates the existence of interactions between $\mathrm{SiO}_{2}$ and water present in the gel.

Analysis of FTIR spectra then agrees with conductivity data. Among the composite membranes the highest conductivity value is, in fact, obtained for the silica containing sample, M4, where interactions between the oxide and polymer components and water retention ability leave the $\mathrm{Li}^{+}$ions available as charge carriers.

Preliminary experiments have been carried out to verify the possibility of application of the composite membranes prepared for EEG recording. All the gel had the mechanical characteristic and ease of manipulation required.

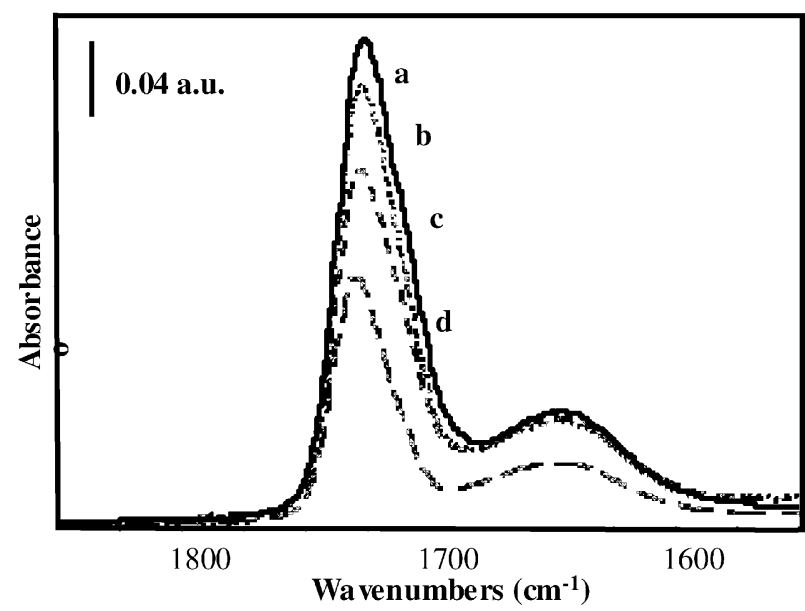

Fig. 3. ATR-FTIR spectra in the $\mathrm{C}=\mathrm{O}$ stretching region of membranes containing $\mathrm{LiClO}_{4} 0.8 \mathrm{M}$ doped with different ceramics: (a) M3, (b) M1, (c) M2 and (d) M4. Labels refer to Table 1.

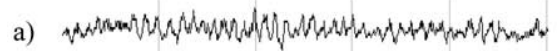

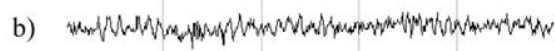

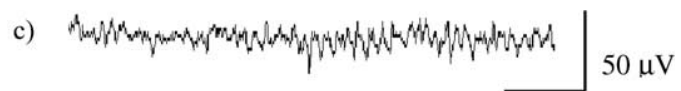

$2 \mathrm{sec}$

Fig. 4. Comparison of bioelectric trace recordings between (a) conventional apparatus and the new polymer gel membranes doped with (b) $5 \mathrm{wt} \% \mathrm{TiO}_{2}$ and (c) $5 \mathrm{wt} \% \mathrm{Al}_{2} \mathrm{O}_{3}$. 
Samples of spontaneous cerebral bioelectrical signals were recorded from healthy humans through M2 and M3 electrodes and were compared to those obtained via conventional dermal electrodes for human (EEG) recording. Fig. 4 shows samples of EEG signals simultaneously recorded by conventional and composite with $\mathrm{TiO}_{2}$ and $\mathrm{Al}_{2} \mathrm{O}_{3}$ electrodes from adjacent scalp locations. The traces obtained using the new solid electrolyte are comparable to those recorded with the conventional fluid electrolyte. The minor differences observed seem to be compatible with routine use.

Further investigations, devoted to the optimization of the performances of composite membranes in the recording of bioelectrical signals, are in progress.

\section{Conclusions}

The new ionoconducting composite membranes developed showed excellent electrochemical and mechanical stability for a long period of time. ATRFTIR studies allowed us to establish that the observed differences in conductivity are related to the interactions occurring between the polymer and/or lithium ions within the surface of the oxides.

Preliminary experiments on EEG recording demonstrate the feasibility of measuring bioelectrical signals with these new solid gel electrolytes.

The electrical features described make the composite electrolyte membranes very interesting substrates for the development of newly designed gels for biomedical applications.

\section{References}

1. Bidoli, V., Casolino, M., De Pascale, M. P., Furano, G., Morselli, A., Narici, L., Picozza, P., Reali, E., Sparvoli, R., Galper, A. M., Ozerov, Yu.V., Popov, A. V., Vavilov, N. R., Alexandrov, A. P., Avdeev, S. V., Baturin, Yu., Budarin, Yu., Padalko, G., Shabelnikov, V. G., Barbiellini, G., Bonvicini, W., Vacchi, A., Zampa, N., Mazzenga, G., Bartalucci, S., Mazzenga, G., Ricci, M., Adriani, O., Spillantini, P., Boezio, M., Carlson, P., Fuglesang, C., Castellini, G. and Sannita, W., Adv. Space Res., 2000, 25, 2075.

2. Romagnoli, P., Di Vona, M. L., Narici, L., Sannita, W. G., Traversa, E. and Licoccia, S., J. Electrochem. Soc., 2001, 148(12), J63.

3. Gasser, B., Int. J. Care Injured, 2000, 31, S-D48.

4. Ramakrishna, S., Mayer, J., Wintermantel, E. and Leong, Kam W., Comp. Science Tech., 2001, 61, 1189.

5. Orthopedic composites. In International Encyclopedia of Composites, ed. Stuart M. Lee. VCH Publishers, New York, 1991. Vol. 4, pp.74-87.

6. Hague, D. C. and Majo, M. J., J. Am. Ceram. Soc., 1994, 77, 1957.

7. Carotta, M. C., Butturi, M. A., Martinelli, G., Di Vona, M. L., Licoccia, S. and Traversa, E., Electron. Technology, 2000, 33, 113.

8. Appetecchi, G. B., Romagnoli, P. and Scrosati, B., Electrochem. Comm., 2001, 3, 281.

9. Trombetta, M., Busca, G., Rossigni, S., Piccoli, V., Corsaro, U., Guercio, A., Catani, R. and Willey, R. J., J. Catal., 1998, 179, 581.

10. Chen, H.-W., Lin, T.-P. and Chang, F.-C., Polymer, 2002, 43, 5281. 